\title{
COMPACT LORENTZIAN MANIFOLDS WITHOUT CLOSED NONSPACELIKE GEODESICS
}

\author{
GREGORY J. GALLOWAY
}

\begin{abstract}
We prove that every compact two-dimensional Lorentzian manifold contains a closed timelike or null geodesic. We then construct a two-dimensional example without any closed timelike geodesics and a three-dimensional example without any closed timelike or null geodesics.
\end{abstract}

An elementary result of causal theory asserts that every compact Lorentzian manifold contains a closed timelike curve $\gamma$. In [2] the author established sufficient conditions for being able to deform $\gamma$ through timelike curves to a closed timelike geodesic. Previously, Tipler [8] had given somewhat more specialized criteria for the existence of a closed timelike geodesic. Neither of these results settles the question: Does every compact Lorentzian manifold contain a closed timelike geodesic? An answer in the affirmative would provide an analogue to the well-known Riemannian result that every compact Riemannian manifold contains a closed geodesic. In this note we prove the following

THEOREM. Every compact two-dimensional Lorentzian manifold contains a closed timelike or null geodesic.

We then construct a two-dimensional example without any closed timelike geodesics and a three-dimensional example without any closed timelike or null geodesics. The more general question as to whether every compact Lorentzian (or pseudoRiemannian) manifold contains a closed geodesic is, as far as we know, still open. (The examples alluded to above have closed spacelike geodesics.)

PROOF OF THE THEOREM. The proof makes use of some standard concepts and results from the causal theory of Lorentzian manifolds for which $[\mathbf{5}, \mathbf{6}, \mathbf{7}, \mathbf{1}]$ are excellent references.

Let $M^{2}$ be a smooth compact manifold equipped with a Lorentzian metric $g$. By taking an appropriate finite covering of $M^{2}$ we can assume $M^{2}$ is orientable and time-orientable. Since $M^{2}$ admits a Lorentzian metric it must have vanishing Euler characteristic, and hence is diffeomorphic to the 2-torus. Since every compact Lorentzian manifold contains a closed timelike curve, consideration of the space-time $\left(M^{2},-g\right)$ shows that $\left(M^{2}, g\right)$ contains a smooth compact spacelike hypersurface $\Sigma$, which is the image of a smooth imbedding of $S^{1}$.

We take a moment to observe that $\Sigma$ does not separate $M^{2}$. Suppose to the contrary that it did. Then $\Sigma$ bounds in $M^{2}$ and hence the homology class determined by $\Sigma$ is trivial in $H_{1}\left(M^{2}, Z\right)$. Since $\Pi_{1}\left(M^{2}\right)$ is abelian, there is a standard isomorphism between $H_{1}\left(M^{2}, Z\right)$ and $\Pi_{1}\left(M^{2}\right)$ which shows that $\Sigma$ is homotopic to a point. It follows that any component $\tilde{\Sigma}$ of $p^{-1}(\Sigma)$, where $\left(\tilde{M}^{2}, p\right)$ is the universal covering

Received by the editors September 10, 1985.

1980 Mathematics Subject Classification (1985 Revision). Primary 53C50; Secondary 53C22. 
manifold of $M^{2}$, is a simple closed curve in $\tilde{M}^{2}$. Since $M^{2}$ is time-orientable it admits a smooth nonvanishing timelike vector field $T$. In particular, $T$ is transverse to $\Sigma$. Lift $T$ to a smooth nonvanishing vector field $\tilde{T}$ on $\tilde{M}^{2}$. Since $\tilde{T}$ is nonvanishing, its Kronecker index as a vector field defined along $\tilde{\Sigma} \subset \tilde{M}^{2} \approx \mathbf{R}^{2}$ must be zero. On the other hand, since $\tilde{T}$ is transverse to $\tilde{\Sigma}$ its Kronecker index must be nonzero.

Thus, $\Sigma$ does not separate and hence may not be acausal. We introduce at this point what is known in relativity theory as the Geroch covering manifold of $M^{2}$ relative to $\Sigma[4]$. Denote this covering by $\left(M_{\Sigma}, p\right)$, where $p: M_{\Sigma} \rightarrow M$ is the covering map. $M_{\Sigma}$ becomes a Lorentzian manifold in the lifted metric. We mention here only those properties of $\left(M_{\Sigma}, p\right)$ needed in the proof: (1) $\left(M_{\Sigma}, p\right)$ is a regular covering, (2) each component $\tilde{\Sigma}$ of $p^{-1}(\Sigma)$ is a compact spacelike hypersurface which separates $M_{\Sigma}$, and (3) the sets $\overline{J^{-}(p)} \cap J^{+}(\tilde{\Sigma})$ and $\overline{J^{+}(p)} \cap J^{-}(\tilde{\Sigma})$ are compact for each $p \in M_{\Sigma}$ (this latter property uses the compactness of $M$ ).

Now let $\tilde{\Sigma}$ be a component of $p^{-1}(\Sigma)$. By property (2), $\tilde{\Sigma}$ is acausal. If $\tilde{\Sigma}$ is a Cauchy hypersurface for $M_{\Sigma}$ then the criteria of Tipler [8] for the existence of a closed timelike geodesic in $M^{2}$ are satisfied, and we are done. Suppose then that $\tilde{\Sigma}$ is not Cauchy. In this case $\tilde{\Sigma}$ has a nontrivial Cauchy horizon. Suppose without loss of generality, $H^{+}(\tilde{\Sigma}) \neq \varnothing$. Let $\eta:[0, a) \rightarrow M_{\Sigma}$ be a past inextendible null geodesic generator of $H^{+}(\tilde{\Sigma})$. Let $\left\{a_{n}\right\}$ be a sequence of nonnegative numbers such that $a_{n} \uparrow a$, and let $q_{n}=\eta\left(a_{n}\right)$. By property (3) above, $\left\{q_{n}\right\}$ is contained in a compact set and hence, by passing to a subsequence if necessary, we can assume $q_{n} \rightarrow q \in M_{\Sigma}$. Let $\gamma$ be a timelike geodesic segment which passes through $q$. From the two dimensionality, there exists a geodesically convex neighborhood $U$ of $q$ such that the null geodesics in $U$, when appropriately parameterized, arise as the integral curves of two pointwise linearly independent smooth null vector fields on $U$. Since $\gamma$ is transverse to these curves, it follows that null geodesics which come close to $q$ must meet $\gamma$. Consequently, $\eta$ will meet $\gamma$ for infintely many parameter values. This leaves only two possibilities: either $\eta$ is a closed null geodesic, or distinct points of $\eta$ can be joined by a timelike curve. However, this latter possibility is ruled out by the fact that $H^{+}(\tilde{\Sigma})$ is achronal. Thus, $p \circ \eta$ is a closed null geodesic in $M$. This concludes the proof.

We now give an example of a two-dimensional compact space-time $\left(V^{2}, g\right)$ without closed spacelike geodescis. (By convention, spacelike vectors have positive square norm.) Then $\left(V^{2},-g\right)$ will be a compact space-time without closed timelike geodesics.

EXAMPLE. Let $M^{2}$ be $\mathrm{R}^{2}$ equipped with the Lorentzian metric,

$$
d s^{2}=\cos ^{2} x\left[d x^{2}-d y^{2}\right]+2 \sin x d x d y,
$$

where $(x, y)$ are cartesian coordinates. This space-time is depicted in Figure 1 along with some of its null cones and geodesics. The metric (1) is invariant under the translations $x \rightarrow x+2 \pi, y \rightarrow y+1$. Let $G$ be the group of isometries generated by these translations. The quotient manifold $V^{2}=M^{2} / G$ is a Lorentzian surface diffeomorphic to the 2-torus.

Suppose $s \rightarrow \sigma(s), 0 \leq s \leq L$, is a closed spacelike geodesic in $V^{2}$ parameterized with respect to arc length. Let

$$
\tilde{\sigma}: x=x(s), \quad y=y(s), \quad 0 \leq s \leq L,
$$




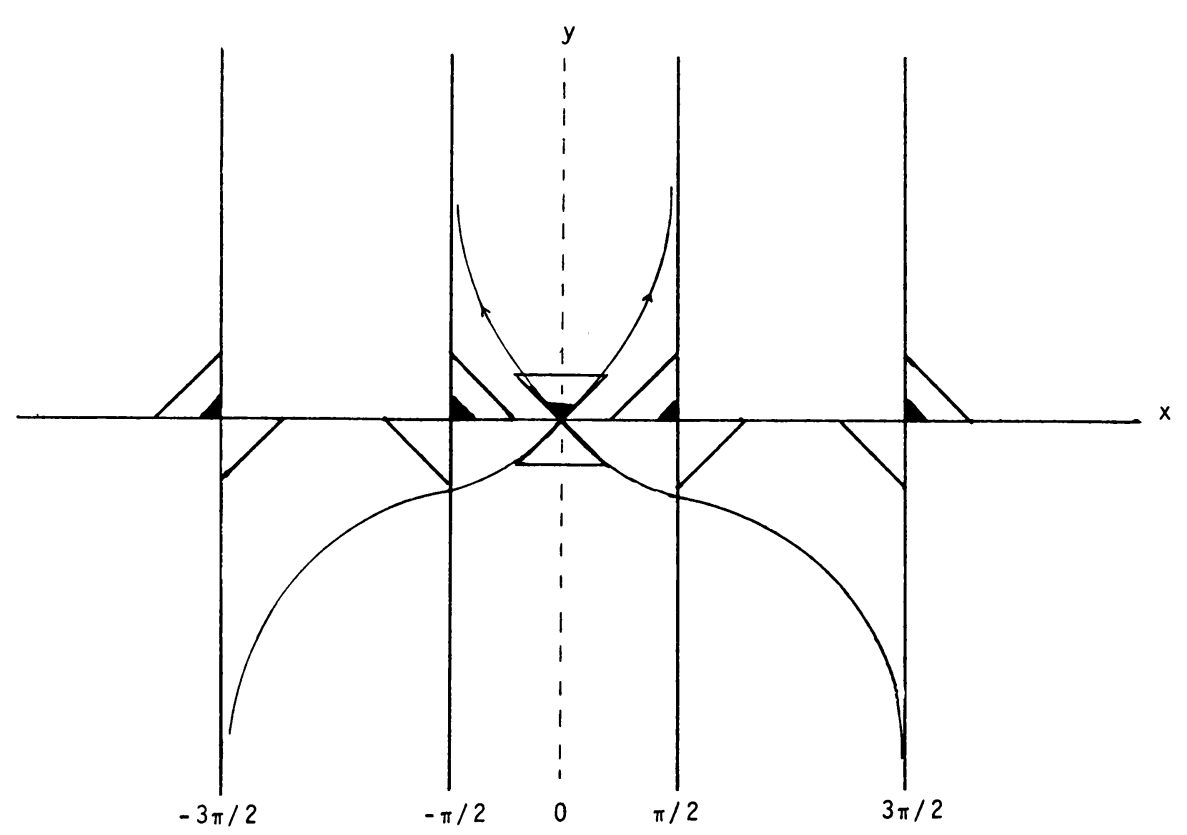

FIGURE 1. The diagram depicts the space-time $M^{2}=\mathbf{R}^{2}$ equipped with the Lorentz metric $d s^{2}=\cos ^{2} x\left[d x^{2}-d y^{2}\right]+2 \sin x d x d y$.

be a lift of $\sigma$ into $M^{2}$. By the discussion at the beginning of the proof of the preceding theorem, $\tilde{\sigma}$ is not closed. This can be seen directly as follows. If $\tilde{\sigma}$ is closed then $x^{\prime}\left({ }^{\prime}=d / d s\right)$ must vanish for some value of $s$. The assumption that $\tilde{\sigma}$ is spacelike then implies the equation $\left(\cos ^{2} x\right)\left(y^{\prime}\right)^{2}=-1$ at this value, which is impossible. Thus, $x^{\prime}$ does not vanish, and hence $\tilde{\sigma}$ can be oriented so that $x^{\prime}>0$ on $[0, L]$. It follows that $x(s)$ takes on all values in an interval of the form $\left[x_{0}, x_{0}+2 n \pi\right]$ for some positive integer $n$. Let $s_{-}, s_{+}$be values in $[0, L]$ such that $x\left(s_{ \pm}\right)= \pm \pi / 2$ $\bmod 2 \pi$.

The coordinate vector $\partial / \partial y$ is a Killing vector field on $M^{2}$. Hence, by the constant of motion lemma (see e.g. $[6]), g\left(\partial / \partial y, \tilde{\sigma}^{\prime}\right)=K=$ constant along $\tilde{\sigma}$, which using (1) gives

$$
-\left(\cos ^{2} x\right) y^{\prime}+(\sin x) x^{\prime}=K
$$

Plugging in the values $s=s_{-}, s_{+}$yields

$$
x^{\prime}\left(s_{+}\right)=K=-x^{\prime}\left(s_{-}\right),
$$

which implies that $K$ changes sign, an impossibility.

Thus $V^{2}$ contains no closed spacelike geodesics. In fact, a closer analysis shows that if $\gamma$ is a spacelike geodesic in $M^{2}$ whose tangent points upward and to the right at the point where $\gamma$ crosses $x=0$ then $\gamma$ approaches $x=\pi / 2$ asymptotically when extended indefinitely in the direction of its tangent. If the tangent points downward and to the right then $\gamma$ approaches $x=-\pi / 2$ asymptotically when extended in the opposite direction. One can also show that all such geodesics are incomplete except for the one that crosses $x=0$ horizontally.

According to the theorem, $V^{2}$ must contain closed null geodesics. Indeed, the lines $x= \pm \pi / 2$ when suitably parameterized, project to closed null geodesics in 
$V^{2}$. It is easily checked that these are the only closed null geodesics. We now use a trick of Carter [5, p. 195] which, by jacking the dimension up by one, enables us to eliminate the closed null geodesics, as well.

EXAMPLE. Let $N^{3}$ be $\mathbf{R}^{3}$ equipped with the metric,

$$
d s^{2}=-\alpha\left(d x^{2}-d y^{2}\right)-2 \beta d x d y+d z^{2},
$$

where $\alpha(x)=\cos ^{2} x$ and $\beta(x)=\sin x$. Note that $N^{3}$ is the geometric product of $M^{2}$ from the previous example (but with metric of opposite sign) and the standard (positive definite) real line. Let $a$ be an irrational number between zero and one. Let $H$ be the group of isometries generated by the maps: $x \rightarrow x+2 \pi, z \rightarrow z+1$, and $(y, z) \rightarrow(x, y+1, z+a)$, and let $W^{3}=N^{3} / H$. Thus, as a manifold we can view $W^{3}$ as the box: $0 \leq x \leq 2 \pi, 0 \leq y \leq 1,0 \leq z \leq 1$ with opposite faces identified as follows: $(0, y, z) \sim(2 \pi, y, z),(x, y, 0) \sim(x, y, 1),(x, 0, z) \sim(x, 1,(z+a) \bmod 1)$.

The argument used in the previous example can be used again to show that there are no closed timelike geodesics in $W^{3}$. We now show that there are no closed null geodesics either. Suppose $s \rightarrow \eta(s), 0 \leq s \leq L$ is a (nontrivial) closed null geodesic in $W^{3}$. Let

$$
\tilde{\eta}: x=x(s), \quad y=y(s), \quad z=z(s), \quad 0 \leq s \leq L,
$$

be a lift of $\sigma$ into $N^{3}$. The constant of motion lemma applied to the Killing fields $\partial / \partial y, \partial / \partial z$, and the assumption that $\tilde{\sigma}$ is null lead to the equations

$$
\begin{gathered}
-\alpha\left(x^{\prime}\right)^{2}+\alpha\left(y^{\prime}\right)^{2}-2 \beta x^{\prime} y^{\prime}+\left(z^{\prime}\right)^{2}=0 \\
\alpha y^{\prime}-\beta x^{\prime}=A \\
z=B s+C
\end{gathered}
$$

where $A, B, C$ are constants.

Suppose $x^{\prime}\left(s_{0}\right)=0$ for some $s_{0} \in[0, L]$. By equation (3), there are two possibilities.

Case 1. $y^{\prime}\left(s_{0}\right)=0$ and $z^{\prime}\left(s_{0}\right)=0$. Since the derivatives of $x, y$, and $z$ all vanish at $s=s_{0}, \eta$ must be trivial by the uniqueness of solutions to the geodesic equation.

Case 2. $\alpha\left(x\left(s_{0}\right)\right)=0$ and $z^{\prime}\left(s_{0}\right)=0$. From equation (5), we have $z=$ constant. From equation (4) we see that $A=0$, and hence $\alpha y^{\prime} \equiv \beta x^{\prime}$. Substitution of these into equation (3) gives $x^{\prime} \equiv 0$ and hence $x=$ constant. Now, because of the irrational translation, projection down into $W^{3}$ of the line $x=$ const., $z=$ const. gives a curve which does not close up.

Thus, we can assume that $x^{\prime}$ never vanishes on the interval $[0, L]$. One can now proceed with the argument used in the timelike case to arrive at a contradiction.

REMARK. This remark refers to the result in [3] that every SCTP (spatially closed time-periodic) space-time $M$ contains a compact spacelike maximal hypersurface. $M$ is said to be spatially closed if it admits a compact spacelike Cauchy hypersurface $S$. According to the definition in [3], $M$ is SCTP if in addition (1) there exists a discrete group of isometries $\psi_{n}: M \rightarrow M, n \in Z$, such that $S_{n} \subset I^{-}\left(S_{n+1}\right)$ and $M=\bigcup_{n \in Z} J^{+}\left(S_{n}\right) \cap J^{-}\left(S_{n}\right)$, where $S_{n}=\psi_{n}(S)$, and (2) for each $p \in S$ there exists a positive integer $n$ such that $p \ll \psi_{n}(p)$.

Let $M^{2}$ be as in the first example above. Let $X^{2}=M^{2} / K$, where $K$ is the group of isometries generated by the translation $x \rightarrow x+2 \pi$. it is easily checked 
that $X^{2}$ is spatially closed. Furthermore, the existence of the Killing field $\partial / \partial y$ shows that the principal time-periodic condition (1) is satisfied. However, the more technical condition (2) does not hold on $X^{2}$ (as can be seen by considering points in $X^{2}$ corresponding to $\left.x= \pm \pi / 2 \bmod 2 \pi\right)$. Since $X^{2}$ does not contain any compact maximal hypersurfaces (these just being closed spacelike geodesics in dimension two), the example illustrates the importance of condition (2).

\section{REFERENCES}

1. J. K. Beem and P. E. Ehrlich, Global Lorentzian geometry, Dekker, New York, 1981.

2. G. J. Galloway, Closed timelike geodesics, Trans. Amer. Math. Soc. 285 (1984), 379-388.

3. 423-429.

4. R. Geroch, Topology in general relativity, J. Math. Phys. 8 (1967), 782-786.

5. S. W. Hawking and G. F. R. Ellis, The large scale structure of the universe, Cambridge Univ. Press, Cambridge, 1973.

6. B. O'Neill, Semi-Riemannian geometry, Academic Press, New York, 1983.

7. R. Penrose, Techniques of differential topology in relativity, SIAM, Philadelphia, Pa.,1972.

8. F. Tipler, Existence of closed timelike geodesics in Lorentz space, Proc. Amer. Math. Soc. 76 (1979), 145-147.

Department of Mathematics, University of Miami, Coral Gables, Florida 33124 